\title{
Resultados de la resonancia magnética nuclear de cerebro en niños con cefalea
}

\author{
Brain magnetic resonance imaging findings in children with \\ headache
}

\author{
Dra. Esra Gurkas, ${ }^{a}$ Dra. Zeynep S. Karalok, ${ }^{a}$ Dra. Birce D. Taskm, ${ }^{a}$ \\ Dra. Ummu Aydogmus, ${ }^{a}$ Prof. Asoc. Cahide Yllmaz y Dra. Gulsah Bayram ${ }^{b}$
}

a. Departamento de Neurología Pediátrica, Hospital de Formación e Investigación en Salud Infantil, Hematología y Oncología de Angora, Angora, Turquía.

b. Departamento de Radiología, Hospital de Formación e Investigación en Salud Infantil, Hematología y Oncología de Angora, Angora, Turquía.

Correspondencia:

Dra. Esra Gurkas: esragurkas@yahoo.com

Financiamiento:

Ninguno.

Conflicto de intereses:

Ninguno que declarar.

Recibido: 4-2-2017

Aceptado: 21-6-2017

\section{RESUMEN}

Introducción: El objetivo fue describir los resultados de la resonancia magnética nuclear (RMN) en niños con cefalea.

Población y métodos: Revisión retrospectiva de las historias clínicas de los pacientes ingresados a los consultorios externos deneurología pediátrica con síntomas de cefalea entre enero de 2013 y diciembre de 2014.

Resultados: Se ingresaron 478 pacientes (273 mujeres, 205 varones) con síntomas de cefalea. Los tipos de cefalea fueron migraña en 218 pacientes $(45,6 \%)$, cefalea tensional en $159(33,3 \%)$, cefalea secundaria en $39(8,2 \%)$ y cefalea inespecífica en $62(13 \%)$. Se realizó una RMN de cerebro a 407 pacientes (85\%); se observaron anomalías cerebrales en 128 pacientes $(31,4 \%)$; cinco tenían anomalías cerebrales relevantes para cefalea, incluso tumores. Entre los otros 123 pacientes, los hallazgos casuales más frecuentes correspondieron a 42 casos $(10 \%)$ de anomalías inespecíficas de la sustancia blanca, 17 casos (4\%) de espacios perivasculares agrandados, 17 casos $(4 \%)$ de quiste aracnoideo, 16 casos $(3,9 \%)$ de ventrículos asimétricos, 12 casos $(2,9 \%)$ de malformación de Chiari tipo 1 y ectopia amigdalina cerebelosa. Asimismo, 17 pacientes $(4,1 \%)$ tenían anomalías extracerebrales en la RMN, entre otras, sinusitis, engrosamiento de la mucosa y quistes de retención de los senos paranasales.

Conclusiones: A pesar del incremento en la realización de estudios de neuroimagenología, la contribución de la RMN de cerebro al diagnóstico y el tratamiento de los niños con cefalea es aún baja.

Palabras clave: cefalea, resonancia magnética nuclear, niño.

http:/ / dx.doi.org/10.5546/aap.2017.e349

Texto completo en inglés:

http:/ / dx.doi.org/10.5546/ aap.2017.eng.e349

Cómo citar: Gurkas E, Karalok ZS, Taskin BD, et al. Resultados de la resonancia magnética nuclear de cerebro en niños con cefalea. Arch Argent Pediatr 2017;115(6):e349-e355.

\section{INTRODUCCIÓN}

La cefalea es un trastorno frecuente en los niños. Según los informes, las tasas de prevalencia de la cefalea durante la niñez varían entre el $26,6 \%$ y el $93,3 \% .{ }^{1,2}$ En un estudio realizado en Turquía, Narlı K. y col. informaron que la prevalencia de la cefalea recurrente en los adolescentes de entre 12 y 17 años era del 52,2\%. ${ }^{3}$

La mayoría de las cefaleas en los niños se debe especialmente a situaciones como las cefaleas primarias. La etiología de la cefalea en los niños y adolescentes puede determinarse principalmente con base en el registro detallado de los antecedentes y en un examen neurológico integral. No se recomienda realizar estudios de neuroimagenología de rutina en los niños con cefalea. El Subcomité de Estándares de Calidad de la Academia Estadounidense de Neurología y el Comité de Práctica de la Sociedad de Neurología Pediátrica publicaron, en 2002, las recomendaciones sobre los estudios de diagnóstico por imágenes en los niños con cefalea. Según estas recomendaciones, entre las indicaciones para la realización de estudios de diagnóstico por imágenes del cráneo se incluyen cefalea aguda intensa, examen neurológico anormal, cambios en el patrón de la cefalea, signos de aumento de la presión intracraneal y coexistencia de convulsiones. ${ }^{4}$ Sin embargo, en la práctica clínica, los estudios de neuroimagenología suelen solicitarse durante la evaluación inicial de los niños con cefalea debido al temor de pasar por alto una enfermedad 
grave y las exigencias cada vez mayores de los padres. Con el aumento de la tasa de realización de procedimientos de neuroimagenología, también se ha incrementado la detección casual de anomalías no relacionadas con la cefalea.

En este estudio, nuestro objetivo fue describir los resultados de la resonancia magnética nuclear $(\mathrm{RMN})$ en niños con cefalea.

\section{POBLACIÓN Y MÉTODOS}

Este fue un estudio retrospectivo llevado a cabo en un hospital de alta complejidad en Angora, Turquía. Se incluyó a niños que ingresaron a los consultorios externos de neurología pediátrica con cefalea como el síntoma principal entre enero de 2013 y diciembre de 2014. Se excluyó a los pacientes con lesión intracraneal conocida.

En nuestros consultorios externos de neurología pediátrica se empleó un abordaje estandarizado para los pacientes con cefalea. A todos los niños se los evaluó para obtener una anamnesis minuciosa y se les realizó un examen físico sistémico que incluyó la medición de la presión arterial y una evaluación neurológica y oftalmológica. En cada visita, el neurólogo pediátrico guardó todos los datos en un archivo estructurado. Se realizó la revisión retrospectiva de estos datos, que incluían edad al momento de la presentación, sexo, duración de la enfermedad, frecuencia de los ataques de cefalea, localización, calidad de la cefalea, influencia de la actividad física, factor desencadenante, síntomas neurológicos asociados y resultados de la RMN de cerebro. Se evaluó a los pacientes cada tres meses después del examen inicial. Se incluyó en el estudio a los pacientes que recibieron seguimiento durante al menos 6 meses.

Se clasificaron los tipos de cefalea según la tercera edición (versión beta) de la Clasificación Internacional de Cefaleas. ${ }^{5}$ Todas las cefaleas se dividieron en dos grupos principales: primarias o secundarias. Luego, las cefaleas primarias se dividieron en cuatro grupos: migraña, cefalea tensional, cefalalgias trigeminoautonómicas y otras cefaleas primarias. Los criterios para clasificar las cefaleas primarias se basaron en las características específicas de la cefalea y los síntomas asociados. Si la cefalea no tenía una de las características necesarias para cumplir con todos los criterios de un subtipo de cefalea o con los criterios de otro trastorno de cefalea, esta se clasificaba como probable, por ejemplo, migraña probable o cefalea tensional probable. En este estudio, los pacientes del grupo "probable" se incluyeron en el grupo de diagnóstico definitivo. Si el paciente tuvo una cefalea nueva por primera vez con una estrecha relación temporal con otro trastorno conocido como causante de la cefalea, al paciente se lo diagnosticó con cefalea secundaria. Si una cefalea primaria preexistente se volvió crónica con una estrecha relación temporal con el trastorno causante o si una cefalea primaria preexistente empeoró significativamente con una estrecha relación temporal con un trastorno causante, como la sinusitis, al paciente se lo incluyó en el grupo de cefalea secundaria. Si no era posible clasificar el tipo de cefalea según estos criterios durante la primera evaluación, se la incluyó como cefalea de tipo "inespecífico".

Los estudios de neuroimagenología se realizaron según las recomendaciones del Subcomité de Estándares de Calidad de la Academia Estadounidense de Neurología y el Comité de Práctica de la Sociedad de Neurología Pediátrica, publicadas en 2002. ${ }^{4}$ Asimismo, también se hicieron estos estudios cuando los pacientes, o los padres, estaban muy preocupados con respecto a la posibilidad de una enfermedad cerebral preexistente grave y no estaban convencidos con la opinión del médico.

El estudio de neuroimagenología preferente para los pacientes con cefalea fue la RMN. Todas las RMN de cerebro se hicieron en un equipo de RMN de 1.5 Tesla (Infinion 1.5T, Philips, Holanda). Se empleó el mismo protocolo estandarizado, que incluía secuencias espín eco ponderadas en T1 adquiridas en los planos frontal, transversal y sagital con un grosor de corte de 4 a $7 \mathrm{~mm}$, secuencias turbo espín eco ponderadas en $\mathrm{T} 2$, secuencias rápidas de inversión-recuperación atenuada de líquidos (FLAIR) ponderadas adquiridas en los planos transversal y frontal con un grosor de corte de $5 \mathrm{a}$ $7 \mathrm{~mm}$ y secuencias de difusión. Se realizaron otras secuencias, como la aplicación de un agente de contraste, venografía por resonancia magnética o angiografía por resonancia magnética, si estaban clínica o radiológicamente indicadas.

Los resultados de la RMN de los pacientes se agruparon, en primera instancia, como normales o anormales. En segundo lugar, los resultados anormales se clasificaron como anomalías relevantes para cefalea o hallazgos casuales.

El análisis estadístico se realizó con el programa SPSS 17.0 (Chicago, IL, EE. UU.). Se emplearon frecuencias y tablas cruzadas para la interpretación de los datos. Para la comparación 
de los grupos, se emplearon la prueba de $\chi^{2}$ de Pearson, la prueba t de Student, el ANOVA unidireccional y el análisis a posteriori. Un valor de $p<0,05$ se consideró significativo en términos estadísticos.

El estudio fue aprobado por el comité de ética local correspondiente al hospital.

\section{RESULTADOS}

En total, ingresaron 478 pacientes (273 mujeres y 205 varones) con síntomas de cefalea entre enero de 2013 y diciembre de 2014 . Se realizó una RMN de cerebro a 407 pacientes $(85 \%)$, que se incluyeron en el estudio. La media de edad al momento de la visita inicial era de $12,28 \pm 3,29$ años (2,6-18 años). La media de duración de la cefalea antes de la primera consulta fue de 14,09 $\pm 11,15$ meses. Los tipos de cefalea fueron migraña en 218 pacientes $(45,6 \%)$, cefalea tensional en $159(33,3 \%)$, cefalea secundaria en $39(8,2 \%)$ y cefalea inespecífica en $62(13 \%)$. No se observaron diferencias en la media de edad de los pacientes con cefalea primaria y los pacientes con cefalea secundaria $(12,64 \pm 3,14$ años en los pacientes con cefalea primaria, 11,90 $\pm 3,36$ años en los pacientes con cefalea secundaria; $p=0,166$ ). Tampoco se notaron diferencias en la distribución de sexos entre los grupos de cefalea primaria y secundaria $(p=0,47)$. La media de duración de la cefalea antes de la primera consulta era significativamente menor en los pacientes con cefalea secundaria en comparación con los que tenían cefalea primaria $(14,47 \pm 11,3$ meses en los pacientes con cefalea primaria, 10,3 $\pm 8,5$ meses en los pacientes con cefalea secundaria; $p=0,031$ ).
En general, a 407 pacientes (85\%) se les realizó una RMN de cerebro; esta mostró anomalías en $128(31,4 \%)$. Las anomalías en la RMN de cerebro se dividieron en dos categorías: 1) anomalías relevantes para cefalea, como tumores, 2) anomalías casuales. Cinco pacientes tenían anomalías relevantes para cefalea. En la RMN se observó que estos pacientes tenían una lesión expansiva (ocupante de espacio). Fueron evaluados por neurocirujanos y sometidos a cirugía. El primer paciente tenía un tumor glial bien diferenciado frontal (Figura 1-A); el segundo, un tumor neuroectodérmico primitivo (Figura 1-B); y el tercero, un meduloblastoma (Figura 1-C). El cuarto paciente tenía un craneofaringioma; el quinto también tenía un tumor glial bien diferenciado.

Entre los otros 123 pacientes, los resultados casuales más frecuentes correspondieron a 42 casos $(10 \%)$ de anomalías inespecíficas de la sustancia blanca, 17 casos $(4 \%)$ de espacios perivasculares agrandados (Figura 2- $A$ ), 17 casos (4\%) de quiste aracnoideo (Figura 2-B), 16 casos $(3,9 \%)$ de ventrículos asimétricos, 12 casos $(2,9 \%)$ con malformación de Chiari tipo 1 (Figura 2-C) y ectopia amigdalina cerebelosa. Los otros 19 pacientes tuvieron anomalías en la RMN: $5(1 \%)$, agrandamiento de la cisterna magna (Figura $2-D) ; 3(0,7 \%)$, silla turca parcialmente vacía; $3(0,7 \%)$, quiste y calcificación de los plexos coroideos; $1(0,2 \%)$, anomalías del cuerpo calloso; $2(0,5 \%)$, quiste de la glándula pineal; $1(0,2 \%)$, lipoma; $1(0,2 \%)$, malformación vascular; $1(0,2 \%)$, infarto crónico; $1(0,2 \%)$, leucodistrofia; $1(0,2 \%)$, atrofia del hipocampo.

FIGURA 1. RMN de cerebro de los pacientes con tumores; las flechas de color blanco indican la lesión.

(A) Secuencia axial ponderada en T2 donde se observa un tumor glial bien diferenciado en el lóbulo frontal derecho

(B) Secuencia coronal ponderada en T2 donde se observa un tumor neuroectodérmico primitivo en el lóbulo temporal derecho

(C) Imagen axial ponderada en $T 2$ donde se observa un meduloblastoma en el cerebelo
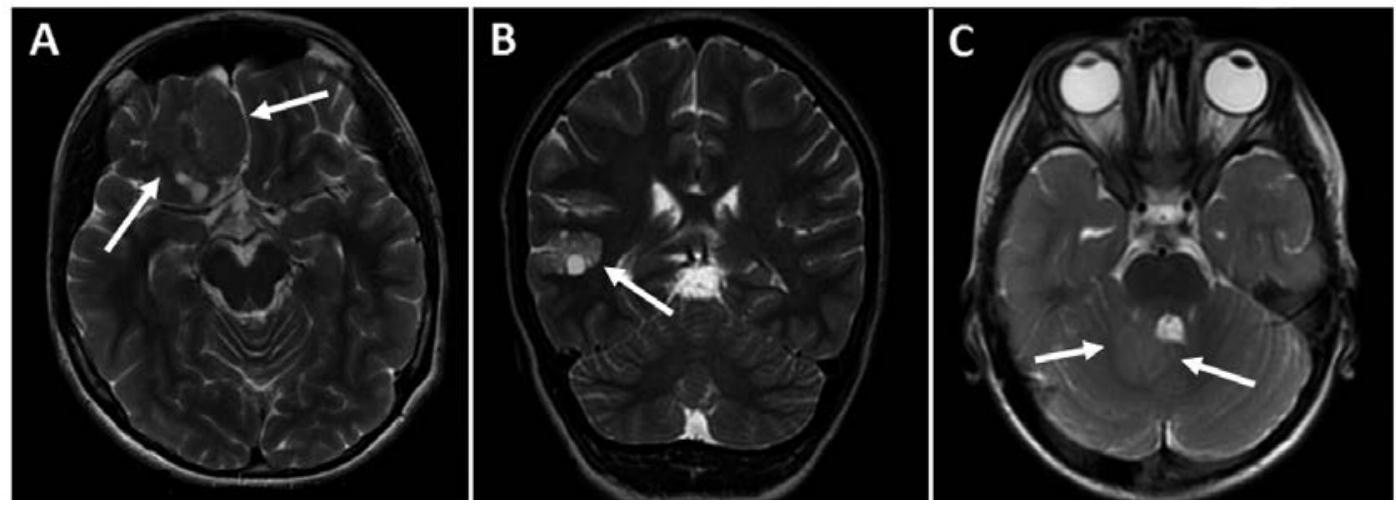
La cefalea en los pacientes con malformación de Chiari tipo 1 y ectopia amigdalina cerebelosa se localizaba, en general, en el lóbulo temporal o frontal, duraba entre 30 minutos y dos horas y no estaba desencadenada por tos o la maniobra de tipo Valsalva, por lo que la característica de la cefalea de estos pacientes no coincidía con los criterios de cefalea atribuidos a la malformación de Chiari tipo 1. Todos los pacientes fueron evaluados por neurocirujanos y no requirieron procedimientos neuroquirúrgicos.

En los pacientes con anomalías inespecíficas de la sustancia blanca, se realizaron otras pruebas, por ejemplo, angiografía por resonancia magnética de cerebro, anticuerpos antinucleares, anticuerpos anti-ADN bicatenario y serología viral para descartar otras etiologías posibles de trastorno de la sustancia blanca. A estos pacientes se les realizó seguimiento clínico y radiológico. No se detectó enfermedad específica, por lo que se consideró que estas hiperintensidades de la sustancia blanca carecían de importancia clínica.

La mitad de los pacientes con anomalías inespecíficas de la sustancia blanca tenían migraña, pero no se observaron diferencias entre la frecuencia de las anomalías inespecíficas de la sustancia blanca en los pacientes con migraña y los otros tipos de cefalea $(p=0,55)$.

Asimismo, 17 pacientes $(4,1 \%)$ tenían anomalías extracerebrales en la RMN, entre otras, sinusitis, engrosamiento de la mucosa y quistes de retención de los senos paranasales. Un otorrinolaringólogo evaluó a estos pacientes. Se confirmó el diagnóstico de sinusitis en 13 pacientes mediante examen clínico y endoscopía nasal. La cefalea en los pacientes con sinusitis estuvo exacerbada por la presión aplicada sobre los senos paranasales y se resolvió significativamente en paralelo con la resolución de la sinusitis con tratamiento. La característica de la cefalea en los pacientes con sinusitis cumple con los criterios de la Clasificación Internacional de Cefaleas para la cefalea atribuida a la sinusitis aguda.

\section{DISCUSIÓN}

La cefalea es un trastorno frecuente en la población pediátrica. La mayoría de los pacientes con cefalea son derivados a los consultorios externos de neurología pediátrica. La migraña y la cefalea tensional son las causas frecuentes de la cefalea en los niños..$^{6-9}$ De manera similar a

FIGURA 2. RMN de cerebro de los pacientes; las flechas de color blanco indican la lesión.

(A) Secuencia axial ponderada en T2 donde se observan espacios perivasculares dilatados

(B) Secuencia axial ponderada en T2 donde se observa un quiste aracnoideo en la región temporal anterior izquierda

(C) Secuencia sagital ponderada en T2 donde se observa agrandamiento de la cisterna magna

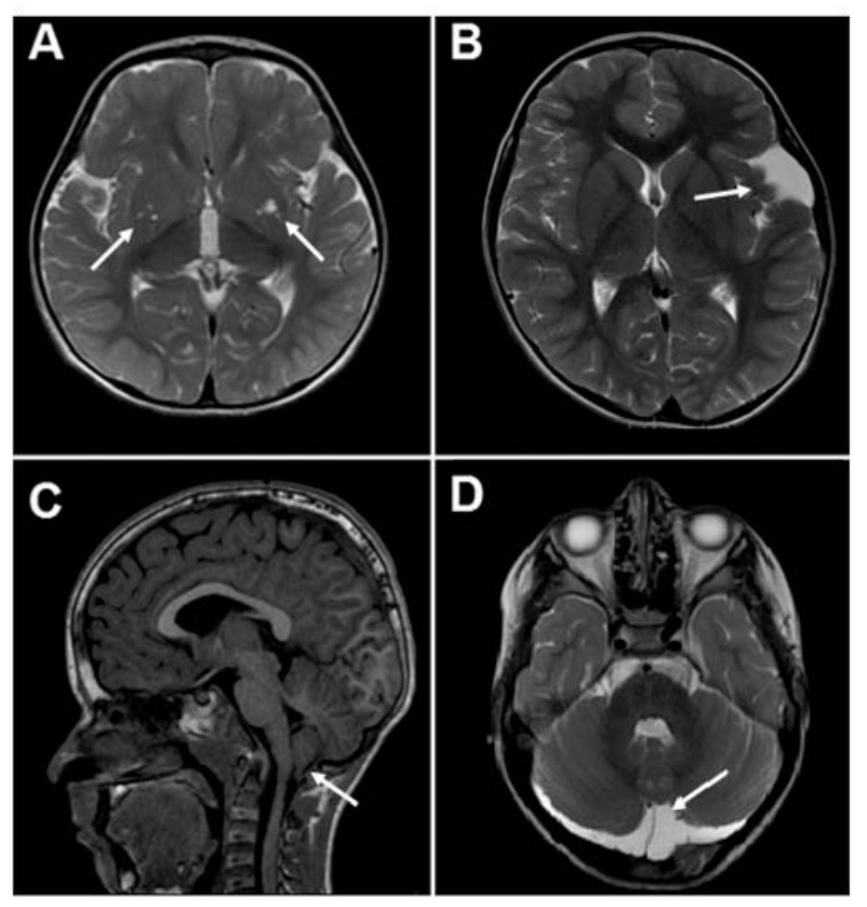


lo observado en las publicaciones científicas, en nuestra serie, la migraña fue el tipo de cefalea más frecuente, seguida de la cefalea tensional. En nuestro estudio, la frecuencia de cefalea secundaria fue del $8,2 \%$, similar a los resultados informados en estudios previos. ${ }^{6-7}$

Según las guías, no se recomienda realizar estudios de neuroimagenología de rutina en niños con cefalea y examen neurológico normal. Sin embargo, en la práctica clínica, los estudios de neuroimagenología suelen solicitarse durante la evaluación inicial de los niños con cefalea debido al temor a pasar por alto una enfermedad preexistente grave y a las mayores exigencias de los padres. En estudios previos, especialmente en los llevados a cabo en los EE. UU., la tasa de estudios de neuroimagenología en los niños con cefalea varía entre el 35,4\% y el 57,6\% . ${ }^{10-12}$ En el estudio actual, se realizaron estudios de neuroimagenología del cerebro en el $85 \%$ de los pacientes. La tasa de neuroimagenología fue mayor en nuestro estudio que en los estudios previos publicados realizados en nuestro país. ${ }^{7-9}$ El porcentaje más elevado podría deberse a las mayores exigencias de los padres y también de los médicos debido al aumento de demandas por negligencia médica, la saturación de las práctica en los consultorios externos y, por lo tanto, el tiempo limitado para realizar una anamnesis y examen físico minuciosos y también los incentivos financieros del seguro médico nacional financiado por el gobierno.

La RMN de cerebro sirve para detectar diversas anomalías en los pacientes con cefalea. Estas anomalías pueden ser relevantes para cefalea y algunas podrían ser lesiones detectadas casualmente. Según los informes, las tasas de anomalías detectadas en los estudios de neuroimagenología de los pacientes pediátricos con cefalea varían entre el 9,3\% y el 21,6\%.6,7,9,10,13 Con el uso actual de las secuencias en los niños con cefalea, la tasa de anomalías detectadas aumentó al 52,8\%, pero el beneficio clínico de estas secuencias sigue siendo limitado. ${ }^{14}$ En nuestro estudio, se detectaron anomalías en la RMN de cerebro del 35\% de los pacientes.

La enfermedad de los senos paranasales fue la principal causa de cefalea en los pacientes con cefalea secundaria. En nuestro estudio, se halló esta enfermedad en 13 niños $(3,1 \%)$ sometidos a estudios de diagnóstico por imágenes para la cefalea. Anteriormente, se informó que la frecuencia de la enfermedad de los senos paranasales era del 1,3\% al 13,7\% en los pacientes sometidos a estudios de diagnóstico por imágenes para la cefalea. ${ }^{10,12,15}$ Esto coincide con nuestros resultados.

La cefalea fue el síntoma principal en el 17\% al $41 \%$ de los pacientes con tumores, aunque la cefalea suele ocurrir con otros síntomas, como vómitos, problemas conductuales y de aprendizaje, inestabilidad y problemas visuales. ${ }^{16-19}$ Aproximadamente el $10 \%$ de los niños con tumores cerebrales tienen un examen físico normal y la cefalea es el único síntoma. ${ }^{6}$ En un metanálisis se revisaron 16 estudios donde se incluyó a 19559 personas sin síntomas neurológicos o psiquiátricos sometidos a una RMN de cerebro como casos de investigación o controles o participantes de una selección comercial, clínica u ocupacional, solo en 135 personas $(0,70 \%)$ se detectaron tumores de manera casual. ${ }^{19}$ En nuestra serie, se detectaron tumores cerebrales en cinco niños $(1,2 \%)$ sometidos a una RMN por cefalea. Los exámenes neurológico y sistémico de estos cinco niños eran normales.

La malformación de Chiari tipo 1 es una protrusión de las amígdalas cerebelosas de $>5 \mathrm{~mm}$ por debajo del agujero magno. La protrusión de las amígdalas cerebelosas de $>5 \mathrm{~mm}$ por debajo del agujero magno se define como ectopia amigdalina cerebelosa. ${ }^{20}$ Los pacientes con malformación de Chiari tipo 1 pueden tener cefalea, parálisis de pares craneales bajos, debilidad y escoliosis. ${ }^{21} \mathrm{En}$ nuestro estudio, el patrón de la cefalea en los pacientes con malformación de Chiari tipo 1 y ectopia amigdalina cerebelosa no fue atribuible a la malformación de Chiari tipo 1. Se halló malformación de Chiari tipo 1 y ectopia amigdalina cerebelosa en el 2,9\% de los pacientes sometidos a una RMN de cerebro. De manera similar, en los estudios previos en niños con cefalea, se observó malformación de Chiari tipo 1 en el 2,7\% al 5,8\% de los participantes. . $^{10,12,22}$ La prevalencia de la malformación de Chiari tipo $1(1,7 \%)$ y de ectopia amigdalina cerebelosa $(0,8 \%)$ fue también del $2,5 \%$ en los pacientes pediátricos atendidos en los consultorios externos de neurología. ${ }^{20}$

Los quistes aracnoideos son bolsas llenas de líquido cefalorraquídeo dentro de la membrana aracnoidea. La mayoría de los quistes aracnoideos son congénitos pero podrían desarrollarse de manera secundaria a un traumatismo, tumor o infección. Los quistes aracnoideos son mayormente asintomáticos pero, en ocasiones, 
pueden causar síntomas debido a la compresión y el sangrado. ${ }^{23}$ En este estudio, se halló un quiste aracnoideo en el $4 \%$ de los pacientes. La prevalencia de los quistes aracnoideos en los estudios previos sobre la cefalea varía entre el $1 \%$ y el $4,4 \% .^{10,12,13,24}$ En este estudio, los estudios de diagnóstico por imágenes de los pacientes con quistes aracnoideos mostraron solo una masa leve y los síntomas de la cefalea no se consideraron relacionados con el quiste.

Los espacios de Virchow-Robin son prolongaciones de la piamadre, pared que rodea las arterias cerebrales. Los espacios de VirchowRobin son estructuras anatómicas normales del sistema nervioso central, en general inferiores a $2 \mathrm{~mm}$ aunque a veces pueden estar dilatados. En nuestra serie, se hallaron espacios de VirchowRobin dilatados en el $4 \%$ de los pacientes sometidos a estudios de diagnóstico por imágenes para la cefalea. De manera similar, Biedron A. y col. evaluaron 1348 RMN de pacientes pediátricos hospitalizados en el departamento de neurología pediátrica y observaron una incidencia de espacios de Virchow-Robin dilatados del 3,93\% . ${ }^{25}$ Asimismo, en el estudio de Gupta y col., la incidencia de espacios perivasculares agrandados fue del $3,8 \% .^{20}$

Las anomalías inespecíficas de la sustancia blanca son focos de desmielinización isquémica y gliosis. ${ }^{26}$ Estas anomalías se detectan mejor como hiperintensidades en las secuencias ponderadas en T2. En este estudio, se hallaron anomalías inespecíficas de la sustancia blanca en 42 de los 127 pacientes (10\%) sometidos a una RMN de cerebro. En estudios previos, la tasa de anomalías de la sustancia blanca variaron entre el 2,9\% y el $4,4 \% \cdot{ }^{7,8,10}$ Nuestro resultado fue más elevado que la tasa publicada anteriormente. Además, varios estudios en niños y adultos indican que los pacientes con migraña tienen un mayor riesgo de anomalías de la sustancia blanca en la RMN de cerebro que los pacientes sin migraña. ${ }^{8,24,27} \operatorname{Sin}$ embargo, aún se desconoce la etiología de las anomalías de la sustancia blanca en los pacientes con migraña. Asimismo, en este estudio, no fue posible establecer una diferencia entre la frecuencia de las anomalías inespecíficas de la sustancia blanca en los pacientes con migraña y con otros tipos de cefalea.

\section{CONCLUSIÓN}

En la práctica clínica, es muy común la realización de estudios de neuroimagenología para el diagnóstico de la cefalea en los niños y adolescentes. Con el aumento de la utilidad de estos estudios, se identificó una tasa elevada de anomalías casuales en los niños con cefalea. La mayoría de estas anomalías que se detectaron de manera casual no estaban asociadas a la cefalea y no contribuyeron al tratamiento y control de esta. Además, estas anomalías casuales también pueden causar una mayor preocupación en los niños y sus padres. Sin embargo, los resultados de estos estudios podrían servir para brindar información a los niños que presentan estas anomalías y a sus familias a fin de reducir su ansiedad.

\section{REFERENCIAS}

1. Dooley JM, Gordon KE, Wood EP. Self-reported headache frequency in Canadian adolescents: Validation and followup. Headache 2005;45(2):127-31.

2. Rhee H. Prevalence and predictors of headaches in US adolescents. Headache 2000;40(7):528-38.

3. Karli N, Akiş N, Zarifoğlu M, et al. Headache prevalence in adolescents aged 12 to 17: a student-based epidemiological study in Bursa. Headache 2006;46(4):649-55.

4. Lewis DW, Ashwal S, Dahl G, et al. Practice parameter: evaluation of children and adolescents with recurrent headaches: report of the Quality Standards Subcommittee of the American Academy of Neurology and the Practice Committee of the Child Neurology Society. Neurology 2002;59(4):490-8.

5. Headache Classification Committee of the International Headache Society (IHS). The International Classification of Headache Disorders. 3rd ed (beta version). Cephalalgia 2013;33(9):629-808.

6. Rho YI, Chung HJ, Suh ES, et al. The role of neuroimaging in children and adolescents with recurrent headaches-multicenter study. Headache 2011;51(3):403-8.

7. Yılmaz Ü, Çeleğen M, Yılmaz TS, tal. Childhood headaches and brain magnetic resonance imaging findings. Eur $J$ Paediatr Neurol 2014;18(2):163-70.

8. Bayram E, Topcu Y, Karaoglu P, et al. Incidental white matter lesions in children presenting with headache. Headache 2013;53(6):970-6.

9. Alehan FK. Value of neuroimaging in the evaluation of neurologically normal children with recurrent headache. J Child Neurol 2002;17(11):807-9.

10. Schwedt TJ, Guo Y, Rothner AD. "Benign" imaging abnormalities in children and adolescents with headache. Headache 2006;46(3):387-98.

11. Graf WD, Kayyali HR, Alexander JJ, et al. Neuroimaginguse trends in nonacute pediatric headache before and after clinical practice parameters. Pediatrics 2008;122(5):e1001-5.

12. Lewis DW, Dorbad D. The utility of neuroimaging in the evaluation of children with migraine or chronic daily headache who have normal neurological examinations. Headache 2000;40(8):629-32.

13. Wöber-Bingöl C, Wöber C, Prayer D, et al. Magnetic resonance imaging for recurrent headache in childhood and adolescence. Headache 1996;36(2):83-90.

14. StreibertPF, Piroth W, Mansour M, et al. Magnetic resonance imaging of the brain in children with headache: the clinical relevance with modern acquisition techniques. Clin Pediatr (Phila) 2011;50(12):1134-9.

15. Maytal J, Bienkowski RS, Patel M, et al. The value of brain imaging in children with headaches. Pediatrics 1995;96(3 Pt 1):413-6. 
16. Wilne SH, Ferris RC, Nathwani A, et al. The presenting features of brain tumours: a review of 200 cases. Arch Dis Child 2006;91(6):502-6.

17. Hayashi N, Kidokoro H, Miyajima Y, et al. How do the clinical features of brain tumours in childhood progress before diagnosis? Brain Dev 2010;32(8):636-41.

18. Nelson S, Taylor LP. Headaches in brain tumor patients: primary or secondary? Headache 2014;54(4):776-85.

19. Morris Z, Whiteley WN, Longstreth WT Jr, et al. Incidental findings on brain magnetic resonance imaging: systematic review and meta-analysis. BMJ 2009;339:b3016.

20. Gupta SN, Belay B. Intracranial incidental findings on brain MRimages in a pediatric neurology practice: a retrospective study. J Neurol Sci 2008;264(1-2):34-7.

21. Greenlee JD, Donovan KA, Hasan DM, et al. Chiari I malformation in the very young child: the spectrum of presentations and experience in 31 children under age 6 years. Pediatrics 2002;110(6):1212-9.

22. Medina LS, Pinter JD, Zurakowski D, et al. Children with headache: clinical predictors of surgical spaceoccupying lesions and the role of neuroimaging. Radiology 1997;202(3):819-24.

23. Gosalakkal JA. Intracranial arachnoid cysts in children: a review of pathogenesis, clinical features, and management. Pediatr Neurol 2002;26(2):93-8.

24. Eidlitz-Markus T,Zeharia A, Cohen YH, etal.Characteristics and management of arachnoid cyst in the pediatricheadache clinic setting. Headache 2014;54(10):1583-90.

25. Biedroń A, Steczkowska M, Kubik A, et al. Dilatation of Virchow-Robin spaces in children hospitalized at pediatric neurology department. Neurol Neurochir Pol 2014;48(1): 39-44.

26. Candee MS, McCandless RT, Moore KR, et al. White matter lesions in children and adolescents with migraine. Pediatr Neurol 2013;49(6):393-6.

27. Swartz RH, Kern RZ. Migraine is associated with magnetic resonance imaging white matter abnormalities: a metaanalysis. Arch Neurol 2004;61(9):1366-8. 